ORIGINAL ARTICLE

\title{
Flucloxacillin alone or combined with benzylpenicillin to treat lower limb cellulitis: a randomised controlled trial
}

\author{
P Leman, D Mukherjee
}

Emerg Med J 2005;22:342-346. doi: 10.1136/emj.2004.019869

\begin{abstract}
Objective: To determine whether using intravenous benzylpenicillin in addition to intravenous flucloxacillin would result in a more rapid clinical response in patients with lower limb cellulitis.

Methods: This was a randomised controlled trial set in an inner city teaching hospital, comprising 81 patients with lower limb cellulitis requiring intravenous antibiotics. The main outcome measure was the mean number of doses of antibiotic required until clinical response.

Results: The mean number of doses required was 8.47 (95\% confidence interval (Cl) 7.09 to 9.86 ) in the benzylpenicillin and flucloxacillin combined group. In the flucloxacillin only group it was 8.71 doses $195 \%$ $\mathrm{Cl} 6.90$ to 10.5$)$, a mean difference of -0.24 doses $(95 \% \mathrm{Cl}-2.48$ to $2.01, \mathrm{p}=0.83)$. Other markers of treatment efficacy showed no difference between groups at review the following day; temperature decrease (mean difference $-0.07^{\circ} \mathrm{C}, 95 \% \mathrm{Cl}-0.76$ to $0.62, \mathrm{p}=0.84$ ), or diameter decrease of affected area (mean difference $-34 \mathrm{~mm}, 95 \% \mathrm{Cl}-99$ to $31, p=0.30$ ). Patient subjective assessments were also similar between the different drug regimen; improvement on a visual analogue scale of pain/discomfort from admission to first review (mean difference $10 \mathrm{~mm}, 95 \% \mathrm{Cl}-12.6$ to $14.2, \mathrm{p}=0.91$ ) and on second review (mean difference $15 \mathrm{~mm}, 95 \% \mathrm{Cl}-18.6$ to $21.6, p=0.88$ ). Patient overall subjective feelings of improvement on first review $(p=0.32)$ and on second review $(p=0.64)$ were also similar.

Conclusions: This study provides no evidence to support the addition of intravenous benzylpenicillin to intravenous flucloxacillin in the treatment of lower limb cellulitis.
\end{abstract}

See end of article for authors' affiliations

Correspondence to: Dr P Leman, Emergency Department, Royal Perth Hospital, Wellington Street, Perth, WA 6001 , Australia; peter.leman@ health.wa.gov.au

Accepted

8 November 2004

C ellulitis is a superficial spreading infection of the skin Most illness can be treated at home with oral antibiotics $^{12}$; however more severe infection, or infection in specific at risk populations continues to represent a significant proportion of hospital admissions, taking up 360000 bed days per year in England alone, ${ }^{3}$ despite increasing home based intravenous treatment regimens. ${ }^{45}$ Research in the treatment of cellulitis has often focused on assessing various combinations of antibiotics, ${ }^{6-9}$ almost all of which have been found to have similar efficacy to standard regimens. It is the standard regimen that remains debatable, however, and it would seem to vary not only across national borders, but within individual hospitals. ${ }^{1011}$

In the UK it is common practice to prescribe both penicillin (penicillin $\mathrm{V}$ for oral use and benzylpenicillin for intravenous use) and flucloxacillin, ${ }^{12}$ and this is recommended by the commonly used national formulary, ${ }^{13}$ authoritative evidence based texts, ${ }^{14}$ and national practice guidelines. ${ }^{15}$ Despite this, there is a singular lack of evidence to support this regimen, ${ }^{14}$ which seems to be based on the premise that benzylpenicillin is required to treat any streptococcal infection and flucloxacillin to treat any staphylococcal infection. What is forgotten by many clinicians is that flucloxacillin is also effective against streptococci. Flucloxacillin is a semi-synthetic isoxazolyl penicillin that maintains the beta-lactam activity against streptococci but incorporates a penicllinase resistant side chain ${ }^{16}$ to prevent inhibition by staphylococci. ${ }^{17}$ This is recognised by microbiologists, which may explain why opinion varies as to the appropriate approach.

Cellulitis is not always a clear cut disease entity, and may be mistakenly diagnosed for other conditions. ${ }^{18} 19$ Furthermore, there appears to be some crossover between the terms "erysipelas" and "cellulitis", ${ }^{14} 2021$ the former usually reserved for a specifically streptococcal infection with a well demarcated edge, the latter being a more general description of a disease that is produced by invasive bacterial infection associated with local erythema, warmth, pain, and swelling. We elected to use only the term cellulitis in our study, as is usual practice in the UK, although this may have included some patients who elsewhere would have been classified as having erysipelas.

While commonly thought to be caused by either streptococci or staphylococci, ${ }^{22}$ many other bacteria have also been associated with the disease. ${ }^{23} 24$ However, routine investigations such as blood cultures ${ }^{25}$ and wound swabs are rarely helpful in early identification of the responsible organism, and antibiotics need to be chosen to maximise efficacy from the onset.

We wished to determine whether treatment with benzylpenicillin in addition to flucloxacillin would provide an improvement in outcome in the treatment of lower limb cellulitis and thus support current practice.

\section{METHODS}

\section{Patients}

This double blind placebo controlled randomised study was performed in a single urban tertiary emergency department (ED) with an annual census of $>105000$ patients. Any patient presenting to the ED with lower limb cellulitis (either unilateral or bilateral) was considered for inclusion. In order to warrant admission for treatment, the size of the cellulitis had to have an initial diameter of $>100 \mathrm{~mm}$. Patients were excluded if they had any allergy to the study drugs, known renal or hepatic impairment, or a random capillary glucose $>13 \mathrm{mmol} / \mathrm{l}$. Patients were also excluded if they had acute co-existent illness in the affected leg, such as deep venous thrombosis, or wound/abscess requiring operative debridement/repair. Patients were required to be able to communicate in written and verbal English. The study protocol received the approval of the local ethics committee. Baseline

Abbreviations: ED, emergency department; VAS, visual analogue scale 


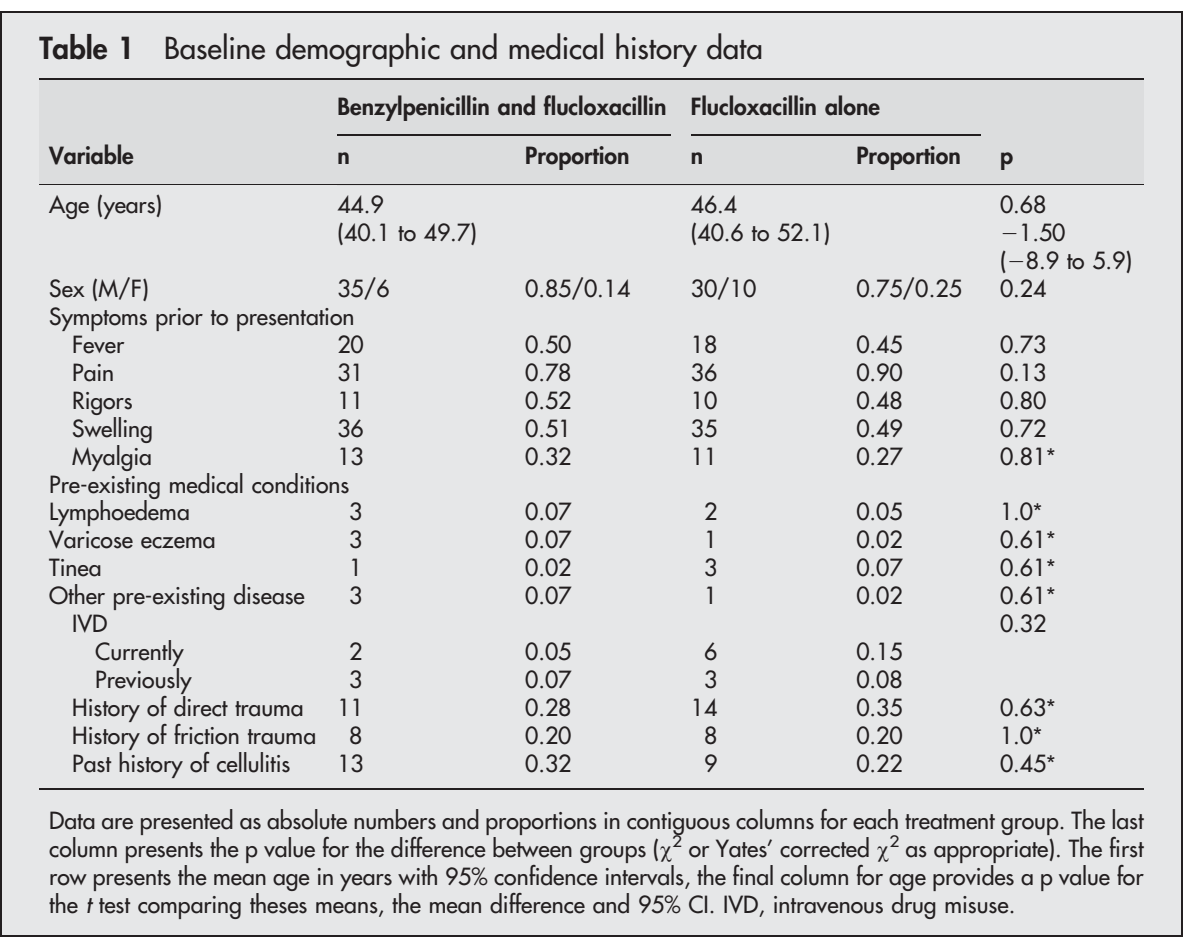

demographic and illness data were collected by the clinician in the ED responsible for their care. All study patients provided written informed consent prior to randomisation.

\section{Main outcome measure}

The main outcome measure chosen was the total number of doses received by the patient prior to clinical response of the disease, pre-defined as both; (a) resolution of the maximal diameter of the cellulitis to either $<100 \mathrm{~mm}$ or $<50 \%$ of the initial diameter and $(b)$ resolution of fever (if present). Patients with an initial diameter between 100 and $200 \mathrm{~mm}$ would achieve clinical response when the diameter decreased to $<100 \mathrm{~mm}$, those with initial diameters $>200 \mathrm{~mm}$ achieved clinical response when the diameter decreased by at least $50 \%$.

\section{Secondary outcome measures}

Secondary disease outcome measures were the level of decrease in fever and decrease in maximum diameter. Patient focused secondary outcome measures were change from baseline on a visual analogue scale (VAS) and overall patient assessment score. Therapy failure rate was also measured. Complications were recorded and patients were free to remove themselves from the study at any time

\section{Interventions and assessments}

After enrolment, the patients were asked to provide scores on a $100 \mathrm{~mm}$ VAS of the pain/discomfort they had in the affected $\operatorname{limb}(\mathrm{s})$. Patients were unaware of their treatment allocation and all were admitted to an emergency observation ward. Enrolled patients had the affected limb elevated and were advised to rest in bed. They all received flucloxacillin $1 \mathrm{~g}$ intravenously four times a day, and were then randomly allocated to receive either benzylpenicillin $1.2 \mathrm{~g}$ intravenously or $10 \mathrm{ml}$ of clear placebo (normal saline). Each of these injections was followed by a standard $10 \mathrm{ml}$ normal saline flush in both groups. Randomisation was performed beforehand using a computer generated sequence, and these were then sealed in opaque envelopes, opened only after the patient was considered eligible for the study. The patients were assessed each morning by an ED clinician who was blinded to treatment allocation. They would assess the appearance of (such as swelling, change from previous day) and measure (using a standard tape measure) the affected area, and check whether the patient was still febrile. They

Table 2 Severity data

\begin{tabular}{|c|c|c|c|c|c|c|}
\hline Variable & $\begin{array}{l}\text { Benzylpenicillin } \\
+ \text { fluclox }\end{array}$ & $95 \% \mathrm{Cl}$ & $\begin{array}{l}\text { Flucloxacillin } \\
\text { alone }\end{array}$ & $95 \% \mathrm{Cl}$ & Difference & $p$ \\
\hline Number of patients & 41 & - & 40 & - & - & - \\
\hline Admission VAS (mm) & 47 & 36 to 58 & 49 & 41 to 58 & $-3(-16$ to 11$)$ & 0.7 \\
\hline $\begin{array}{l}\text { Admission pulse rate } \\
\text { (/min) }\end{array}$ & 94.1 & 87.9 to 100.2 & 90.3 & 84.5 to 96.1 & $3.8(-4.5$ to 12.1$)$ & 0.37 \\
\hline Temperature $\left({ }^{\circ} \mathrm{C}\right)$ & 37.2 & 36.6 to 37.7 & 37.1 & 36.8 to 37.4 & $0.07(-0.55$ to 0.68$)$ & 0.82 \\
\hline Respiration rate (/min) & 15.2 & 14.1 to 16.3 & 16.6 & 15.7 to 17.5 & $-1.40(-2.80$ to 0.00$)$ & 0.05 \\
\hline $\begin{array}{l}\text { Maximum diameter } \\
(\mathrm{mm})\end{array}$ & 267 & 216 to 319 & 200 & 172 to 229 & $667(8.6$ to 125$)$ & 0.2 \\
\hline WCC $\left(\times 10^{9} / \mathrm{I}\right)$ & 10.6 & 8.9 to 12.2 & 11.6 & 10.1 to 13.2 & $-1.1(-3.3$ to 1.2$)$ & 0.34 \\
\hline Urea (mmol/l) & 4.7 & 4.1 to 5.4 & 5.4 & 4.4 to 6.3 & $-0.6(-1.7$ to 0.5$)$ & 0.28 \\
\hline CRP (mgl/l) & 88.1 & 61.2 to 114.9 & 105.0 & 69.9 to 140.1 & $-16.9(-60.3$ to 26.5$)$ & 0.44 \\
\hline ALT (IU/I) & 39.8 & 24.7 to 54.9 & 44.6 & 27.2 to 62.0 & $-4.8(-27.3$ to 17.7$)$ & 0.67 \\
\hline
\end{tabular}


Table 3 Cellulitis outcome

\begin{tabular}{|c|c|c|c|c|c|c|c|c|}
\hline \multirow[b]{2}{*}{ Variable } & \multicolumn{3}{|c|}{ Benzylpenicillin + flucloxacilin } & \multicolumn{3}{|c|}{ Flucloxacillin alone } & \multirow[b]{2}{*}{ Mean difference } & \multirow[b]{2}{*}{$\mathbf{p}$} \\
\hline & Mean & $\mathbf{n}$ & $95 \% \mathrm{Cl}$ & Mean & $\mathbf{n}$ & $95 \% \mathrm{Cl}$ & & \\
\hline $\begin{array}{l}\text { Total no. of doses of } \\
\text { antibiotic received }\end{array}$ & 8.47 & 38 & 7.09 to 9.86 & 8.71 & 38 & 6.90 to 10.5 & $-0.24(-2.48$ to 2.01$)$ & 0.83 \\
\hline $\begin{array}{l}\text { Temperature drop } \\
\text { (day 1-day 0) ( }{ }^{\circ} \mathrm{C} \text { ) }\end{array}$ & 0.36 & 35 & -0.24 to 0.95 & 0.42 & 32 & 0.06 to 0.80 & $-0.07(-0.76$ to 0.62$)$ & 0.84 \\
\hline $\begin{array}{l}\text { Diameter decrease } \\
\text { (day 1-day 0) }(\mathrm{mm})\end{array}$ & 36 & 26 & -20 to 92 & 69 & 22 & 33 to 105 & $-34(-99$ to 31$)$ & 0.30 \\
\hline $\begin{array}{l}\text { Diameter decrease } \\
\text { (day 2-day 0) }(\mathrm{mm})\end{array}$ & 95 & 13 & 35 to 155 & 46 & 12 & -6.0 to 99 & $48(-27$ to 124$)$ & 0.20 \\
\hline
\end{tabular}

would also ask the patient to record a further VAS of their pain, and to answer the overall patient assessment score (a categorical statement as to whether they felt that the cellulitis had either $(a)$ improved, $(b)$ stayed the same, or $(c)$ worsened).

Patients were discharged if the cellulitis met the predefined clinical resolution criteria, otherwise they continued on their current therapy. If the cellulitis edge had advanced after at least 24 hours of intravenous therapy then they were considered a treatment failure and were changed to coamoxiclav $1.2 \mathrm{gm}$ intravenously three times a day.

\section{Post-discharge treatment}

All patients were discharged on oral flucloxacillin $500 \mathrm{mg}$ four times daily for 5 days. They also received penicillin $\mathrm{V}$ $500 \mathrm{mg}$ four times daily if they had been randomised to the intravenous benzylpenicillin group. Placebo penicillin V was not used. Patients were not followed up after discharge from hospital.

\section{Statistical analysis}

We estimated that we would need to recruit a total of 78 patients to have $80 \%$ power to show a clinical significant difference of two doses of antibiotic administered (equivalent to 12 additional hours of treatment). We had an estimated standard deviation of 3.1 doses (determined from retrospective data on previous admissions in our unit) and set alpha at 0.05 . We allowed for $20 \%$ of data being ineligible or unavailable for analysis, thus we planned to recruit 100 patients in total.

Data were analysed with SYSTAT software. We used two tailed $t$ test for continuous data, and $\chi^{2}$ tests for categorical data. Yates' correction was used where appropriate. Where we explored non-equality in baseline variables between groups that may have explained the outcomes measured, we used analysis of covariance to determine the relevant coefficient.

\section{RESULTS}

We recruited a total of 99 patients to the study, of whom 81 were assessable, between September 2001 and March 2003. Among the non-assessable patients were five who were

\begin{tabular}{|c|c|c|c|c|c|}
\hline & \multicolumn{2}{|c|}{$\begin{array}{l}\text { Benzylpen } \\
\text { + fluclox }\end{array}$} & \multicolumn{2}{|c|}{ Fluclox alone } & \multirow[b]{2}{*}{$p\left(\chi^{2}\right.$, d.f } \\
\hline & $\mathrm{n}$ & Prop & $\mathrm{n}$ & Prop & \\
\hline \multicolumn{6}{|l|}{ Day 1} \\
\hline Improving & 25 & 0.74 & 21 & 0.68 & 0.32 \\
\hline No change & 9 & 0.26 & 8 & 0.26 & $(2.27,2)$ \\
\hline Worse & 0 & 0 & 2 & 0.06 & \\
\hline \multicolumn{6}{|l|}{ Day 2} \\
\hline Improving & 18 & 0.82 & 16 & 0.84 & 0.64 \\
\hline No change & 1 & 0.05 & 0 & & $(0.90,2)$ \\
\hline Worse & 3 & 0.14 & 3 & 0.16 & \\
\hline
\end{tabular}

found to be potentially penicillin allergic after randomisation and were removed prior to receiving any medication, two who withdrew consent between randomisation and admission, one who withdrew consent during treatment, three who were admitted directly to an inpatient specialty team and were not available for review on the observation ward, and seven for whom we were unable to either trace the medical record or whose outcome data were missing. There were 41 patients in the combined group (benzylpenicillin and flucloxacillin) and 40 in the flucloxacillin alone group (table 1).

There were no significant differences either in symptoms or in history of potential antecedent risk factors between the two groups (table 2). Fever, pain, and swelling were common symptoms, and half $(41 / 81)$ the patients had a history of some form of trauma prior to presentation. Current or previous illicit intravenous drug use was present in 14 (17\%) of patients. Lymphoedema, varicose eczema, and tinea were uncommonly found.

The patients had no difference in either age or sex and had similar markers of severity except for the maximum diameter of the affected area. This area was greatest in the combined group (2670 mm $v 2000 \mathrm{~mm}$, mean difference $667 \mathrm{~mm}, 95 \%$ confidence interval 86 to $1250 \mathrm{~mm}$ ). The other markers of severity such as pulse rate, respiratory rate and temperature were all very similar. Blood tests performed to measure severity were also similar in both groups, and of note the

Table 4 Patient subjective assessments of outcome (VAS)

\begin{tabular}{|c|c|c|c|c|c|c|c|c|}
\hline \multirow[b]{2}{*}{ Variable } & \multicolumn{3}{|c|}{ Benzylpen + flucloxacillin } & \multicolumn{3}{|c|}{ Flucloxacillin alone } & \multirow[b]{2}{*}{ Mean difference } & \multirow[b]{2}{*}{$\mathbf{p}$} \\
\hline & Mean & $\mathbf{n}$ & $95 \% \mathrm{Cl}$ & Mean & $\mathbf{n}$ & $95 \% \mathrm{Cl}$ & & \\
\hline $\begin{array}{l}\text { VAS change } \\
\text { (day 1-day 0) }\end{array}$ & 2.6 & 24 & 1.6 to 3.6 & 2.5 & 23 & 1.6 to 3.4 & $0.10(-1.26$ to 1.42$)$ & 0.91 \\
\hline $\begin{array}{l}\text { VAS change } \\
\text { (day } 2 \text {-day } 0 \text { ) }\end{array}$ & 3.0 & 16 & 1.4 to 4.7 & 2.9 & 16 & 1.6 to 4.2 & $0.15(-1.86$ to 2.16$)$ & 0.88 \\
\hline
\end{tabular}


mean white cell count was $11.6\left(\times 10^{9} / 1\right)$ in the flucloxacillin alone group compared with $10.6\left(\times 10^{9} / 1\right)$ in the combined group (mean difference $-1.1,-3.3$ to 1.2 ). Similarly, C reactive protein was also similar (105 in the flucloxacillin group $v 88.1$ in the combined group; difference $-16.9 ; 95 \%$ CI -60.3 to 26.5 ) between groups.

The main outcome measure was the total number of doses of antibiotic received until clinical response (table 3). There was no difference between the two groups for this measure (8.47 (7.09 to 9.86) combined group $v 8.71(6.90$ to 10.5$)$ flucloxacillin alone group). Owing to the potential inclusion bias with the significantly larger area of involved limb in the combined group (though with all other markers of severity being similar) we performed an analysis of covariance to account for this potential confounder. We found that the difference in effect was, after taking the size difference into account, equivalent to $-0.81(\mathrm{p}=0.50)$ doses of antibiotic, compared with the mean difference of -0.24 doses before multivariate analysis.

Secondary outcome measures were also similar between the two groups. There was no significant difference in either temperature drop (mean difference $-0.07 ; 95 \%$ CI -0.76 to $0.62)$ or diameter decrease $(-3.4 ;-1.26$ to 1.42$)$ of the cellulitis between the two antibiotic regimen groups. The patient subjective assessments made using VAS and overall scores showed no differences on either day 1 after starting antibiotics or on day 2 (tables 4 and 5).

There were five treatment failures; three received both antibiotics and two received flucloxacillin alone. Four of these patients were changed to co-amoxiclav under the study protocol and one patient had amoxycillin and flucloxacillin. These patients required a mean of 25 doses of antibiotic each, only one grew an organism from blood culture, which was a flucloxacillin sensitive staphylococcus.

There were 66 sets of blood cultures taken on admission; of these 61 had no growth, three grew contaminants and two grew potentially pathogenic organisms (both staphylococci). There were 16 patients with wounds suitable for microbiological swab sampling. Six patients grew Staphylococcus aureus, four grew a streptococcus, and one grew a pseudomonas (although this patient recovered with intravenous flucloxacillin alone). No drug related adverse effects were recorded during the study

\section{DISCUSSION}

We found that there was no clinical difference in outcome when treating lower limb cellulitis with either intravenous flucloxacillin alone or combining it with intravenous benzylpenicillin. Furthermore, there was no difference in subjective outcome from the patient perspective when the two antibiotic regimens were compared. It seems likely from our data that the addition of penicillin to a regimen containing flucloxacillin is unnecessary in the management of cellulitis.

There are potential weaknesses in our findings. These include a large number of randomised patients who did not complete the protocol, albeit that several were inappropriately randomised to begin with (penicillin allergy, admission pending under inpatient team). Our concern over the smaller size of area affected in the flucloxacillin alone group led us to perform the analysis of covariance, on the assumption that this difference allowed flucloxacillin to seem as effective, but only because it was used in patients with milder disease. However, despite this being taken into account in the analysis, the combined regimen was still not more effective. In addition, all other markers of severity, such as $\mathrm{C}$ reactive protein, white cell count, temperature, and patients VAS were similar. In addition, all the outcome measures that we used, both subjective and objective, were very similar for both treatment groups. We used subjective assessments to provide a patient based evaluation of the treatment. We had not previously validated VAS for use in cellulitis and the efficacy of this patient measure is uncertain.

We did not obtain microbiological growth in the majority of our patients, as in most similar studies, but have no reason to suspect that our broad population base differs significantly. We would expect therefore that a range of gram positive organisms were involved, yet all remained sensitive clinically to either of our treatment regimens. The dose of flucloxacillin used is significant in that we would expect high tissue concentrations to achieve the minimum inhibitory concentrations for both staphylococci and streptococci from flucloxacillin alone. We did not see any evidence that benzylpenicillin is more effective against streptococcal infection than flucloxacillin.

Our mean duration of stay to pre-defined clinical resolution was much shorter than that seen in most studies, just over 2 days compared with a range of 5 to 9 days ${ }^{5826}$ and of 8.9 days in routine hospital practice. ${ }^{3}$ This may be that we discharged patients much earlier than in these studies (or in routine practice) and we were unable to determine the exact date of complete resolution of all visible cellulitis. We did not obtain follow up data on our patients, and although we had one patient represent with a recurrence in the study period, others may have been missed or presented to other institutions.

It is unclear whether the high doses we used intravenously can be generalisable to oral dosing for milder disease. It is clear from the literature that several oral dosing regimes are as effective as intravenous regimens across a range of antibiotics ${ }^{27}$ and the necessity for hospital admission could be reduced by choosing outpatient oral or outpatient intravenous treatment. ${ }^{58}$ To determine an appropriate care setting, risk stratification tools are still being developed, although the addition of penicillin seems unnecessary wherever treatment is provided.

\section{Authors' affiliations}

P Leman, D Mukheriee, Emergency Department, St Thomas' Hospital, London, UK

Competing interests: none declared

\section{REFERENCES}

1 Anonymous. Dilemmas when managing cellulitis. Drug Ther Bull 2003;41:43-6.

2 Gentry LR-GG, Zeluff BJ, Khoshdel A, et al. A comparative evaluation of oral ofloxacin versus intravenous cefotaxime therapy for serious skin and skin structure infections. Am J Infect 1989;87:57-60S

3 Department of Health. Hospital episode statistics for England, 2001-2. www.doh.gov.uk/hes/free_data/index.html. Last accessed 15 November, 2003.

4 Grayson ML, Silvers J, Turnidge J. Home intravenous antibiotic therapy. A safe and effective alternative to inpatient care. Med J Aust 1995; 162:249-53.

5 Leder K, Turnidge JD, Grayson ML. Home-based treatment of cellulitis with twice-daily cefazolin. Med J Aust 1998;169:519-22.

6 Bernard P, Chosidow O, Vaillant L. Oral pristinamycin versus standard penicillin regimen to treat erysipelas in adults: randomised, non-inferiority, open trial. BMJ 2002;325:864.

7 Siami G, Christou N, Eiseman I, et al. Clinafloxacin versus piperacillintazobactam in treatment of patients with severe skin and soft tissue infections. Antimicrob Agents Chemother 2001;45:525-31.

8 Grayson ML, McDonald M, Gibson K, et al. Once-daily intravenous cefazolin plus oral probenecid is equivalent to once-daily intravenous ceftriaxone plus oral placebo for the treatment of moderate-to-severe cellulitis in adults. Clin Infect Dis 2002;34:1440-8.

9 Bucko AD, Hunt BJ, Kidd SL, et al. Randomized, double-blind, multicenter comparison of oral cefditoren 200 or $400 \mathrm{mg} \mathrm{BID}$ with either cefuroxime $250 \mathrm{mg}$ BID or cefadroxil $500 \mathrm{mg}$ BID for the treatment of uncomplicated skin and skin-structure infections. Clin Ther 2002;24:1134-47.

10 Dong SL, Kelly KD, Oland RC, et al. ED management of cellulitis: a review of five urban centers. Am J Emerg Med 2001;19:535-40.

11 Aly AA, Roberts NM, Seipol KS, et al. Case survey of management of cellulitis in a tertiary teaching hospital. Med J Aust 1996;165:553-6.

12 Cox NH. Management of lower leg cellulitis. Clin Med 2002;2:23-7. 
13 Medical Association and the Royal Pharmaceutical Society of Great Britain British national formulary, 46th ed, 2003. www.bnf.org/bnf/bnf/current/ doc/102045.htm. Last accessed 15 November 2003.

14 Morris A. Cellulitis and erysipelas. Clin Evid 2002;7:1483-7. Update in: Clin Evid 2003;9:1804-9.

15 Health Protection Agency. Management of infection guidance for primary care for consultation \& local adaptation. London: HPA, 2002. www.hpa.org.uk/infections/topics_az/antibiotic_guidance/ antibiotic_guidance_290404.pdf. Last accessed 15 November, 2003.

16 Sutherland RCE, Rolinson GN. Flucloxacillin, a new isoxazoly penicillin, compared with oxacillin, cloxacillin, and dicloxacillin. BMJ 1970;4:455-60.

17 Rolinson GNSR. Semi-synthetic penicillins. Adv Pharmacol Chemother 1973;11:151-220.

18 Hepburn MJ, Dooley DP, Ellis MW. Alternative diagnoses that often mimic cellulitis. Am Fam Physician 2003;67:2471.

19 Quartey-Papafio CM. Lesson of the week: importance of distinguishing between cellulitis and varicose eczema of the leg. BM 1999;318:1672-3.

20 Dupuy A, Benchikhi H, Roujeau JC, et al. Risk factors for erysipelas of the leg (cellulitis): case-control study. BMJ 1999;318:1591-4.
21 Vaillant L. (Diagnostic criteria for erysipelas). Ann Dermatol Venereol 2001;128:326-33

22 Eriksson B, Jorup-Ronstrom C, Karkkonen K, et al. Erysipelas: clinical and bacteriologic spectrum and serological aspects. Clin Infect Dis 1996:23:1091-8.

23 Gach JE, Charles-Holmes R, Ghose A. E. coli cellulitis. Clin Exp Dermatol 2002;27:523-5.

24 Lee NH, Choi EH, Lee WS, et al. Tuberculous cellulitis. Clin Exp Dermato 2000;25:222-3.

25 Perl B, Gottehrer NP, Raveh D, et al. Cost-effectiveness of blood cultures for adult patients with cellulitis. Clin Infect Dis 1999;29:1483-8.

26 Ramirez-Ronda CHSS, Rivera-Vazquez CR. Comparative, double-blind study of oral ciprofloxacin and intravenous cefotaxime in skin and skin stuctrue infections. Am J Med 1987:82:220-3.

27 Kiani R. Double-blind, double dummy comparison of azithromycin and cephalexin in the treatment of skin and skin structure infections. Eur J Clin Microbiol Infect Dis 1991;10:880-4.

28 Howden BP, Richards MJ. The efficacy of continuous infusion flucloxacillin in home therapy for serious staphylococcal infections and cellulitis. J Antimicrob Chemother 2001;48:311-14. 